\title{
Correspondence
}

\section{Polypharmacy: saint or sinner?}

Lepping \& Harbone ${ }^{1}$ query the notion that polypharmacy rates are increasing. The general consensus, however, is that polypharmacy rates are indeed rising and previous studies clearly report this trend. ${ }^{2,3}$ It is also not certain that the study by Tungaraza et $a l^{4}$ is the first community study of polypharmacy in the UK, as our study ${ }^{5}$ probably predates it.

The findings from our study were strikingly similar to those of Tungaraza et al in showing almost identical out-patient polypharmacy rates of $17.4 \%$ and $17.5 \%$ respectively, and a prevalence of high-dose prescribing and sedative use in association with polypharmacy. These results were obtained despite the fact that our study population would not be considered severely ill. Both studies showed a tendency for atypical antipsychotics to be commonly involved in combination or highdose prescribing - perhaps asking, as do Lepping \& Harbone, about the efficacy of atypicals in the real-life clinical situation.

That polypharmacy continues despite repeated guidance against it may indicate that this is perhaps one area in which clinical practice and observation is ahead of research evidence, which is yet to catch up. Lepping \& Harbone make the point that in the case of polypharmacy the evidence provides no support one way or the other. There appears now, however, to be a shift away from a blanket condemnation of antipsychotic polypharmacy to a search for evidence-based recommendations, which would support a role for polypharmacy in everyday clinical practice. Langan \& Shajahan ${ }^{6}$ provide a number of excellent recommendations based on a thorough review of the existing literature. Not all of these recommendations may, however, be applicable in everyday clinical practice.

Several studies, including ours, ${ }^{5}$ have shown poor adherence to standards requiring documentation of clinical practice, or the recording of investigation reports such as electrocardiograms. Recent audits have advocated review by pharmacists, which may be feasible for in-patients but less so in out-patient populations. It is similarly problematic to conceive of a mechanism to ensure that cross-tapering of medication is completed and not abandoned half-way through. The idea of switching back from polypharmacy to monotherapy in identified cases sounds attractive and has been shown successful in a proportion of patients, ${ }^{6}$ but clinicians may still remain wary of the problem of inducing psychotic relapses in otherwise stable patients, with all the associated consequences, including a fatal outcome.

What is clear perhaps is that the antipsychotic polypharmacy issue is unlikely to go away. The current attempts to 'manage' polypharmacy through audit, guidelines and recommendations have not led to change, and polypharmacy remains in many ways 'treatment resistant'. It may be time to be open-minded about psychiatry's 'dirty little secret' and allow the 'co-prescribing' of new measures focused on achieving a better understanding of the polypharmacy phenomenon.

1 Lepping P, Harborne GC. Polypharmacy: how bad are we really? Psychiatrist 2010; 34: 208-9.
2 Centorrino F, Eakin M, Bahk WM, Kelleher JP, Goren J, Salvatore P, et al. In-patient antipsychotic drug use in 1998, 1993 and 1989. Am J Psychiatry 2002; 159: 1932-5.

3 Gilmer TP, Dolder CR, Folsom DP, Mastin W, Jeste DV. Antipsychotic polypharmacy trends among medical beneficiaries with schizophrenia in San Diego County, 1999-2004. Psychiatr Serv 2007; 58: 1007-10.

4 Tungaraza TE, Gupta S, Jones J, Poole R, Slegg G. Polypharmacy and high-dose antipsychotic regimes in the community. Psychiatrist 2010; 34: 44-6.

5 Ranceva N, Ashraf W, Odelola D. Antipsychotic polypharmacy in outpatients at Birch Hill Hospital: incidence and adherence to guidelines. J Clin Pharmacol 2010; 50: 699-704.

6 Langan J, Shajahan P. Antipsychotic polypharmacy: review of mechanisms, mortality and management. Psychiatrist 2010; 34: 58-62.

Deji Odelola, Consultant Psychiatrist, Birch Hill Hospital, Rochdale, email: deji.odelola@nhs.net, Nadezda Ranceva, Academic Clinical Fellow in Psychiatry (CT1), Meadowbrook Hospital, Greater Manchester West Foundation Trust, Salford.

doi: 10.1192/pb.34.8.354

\section{The jury is still out!}

Lepping \& Harborne ${ }^{1}$ highlight the unfortunate conflation of 'psychotropic polypharmacy' and 'antipsychotic polypharmacy', which is seen in the study by Tungaraza et $a l^{2}$ and which may confuse the reader. Their response falls foul of this issue when they refer to the statement that 'only a third of [patients] were on one psychotropic medication', and draw an implication of a shortfall in compliance with the National Institute for Health and Clinical Excellence schizophrenia guideline. ${ }^{3}$ The guideline advocates sequential use of antipsychotic monotherapy, but does not discuss polypharmacy involving other psychotropic medication. Lepping \& Harborne rightly point out that both Taylor ${ }^{4}$ and Tungaraza et al have made assessments about the temporal change of incidence of antipsychotic polypharmacy without references, but later they mention studies of clozapine-amisulpride and clozapine-quetiapine combinations which are unreferenced.

An internal in-patient survey of antipsychotic polypharmacy in our own trust demonstrated an incidence broadly similar to that found in the literature at the time, but that antipsychotic polypharmacy regimes were not centred around attempts to optimise clozapine treatment. Rather, a variety of regimes involving diverse antipsychotics was seen. It is perhaps speculative to presume that in the Wrexham cohort ${ }^{2}$ most people on two or more antipsychotics were taking clozapine. In the forensic setting, complexity and diagnostic plurality is the norm, so antipsychotic polypharmacy is perhaps unavoidable at times. It is our concern that procedural aspects, such as preconditions for assured concordance before transfer to step-down services, may sometimes colour the prescribing decisions and drive the co-administration of depot antipsychotics with oral atypicals. We could not find reference to non-medical prescribers in Taylor's article. Indeed, we feel that Tungaraza et al suggest that the emergence of new groups of prescribers points out the urgency of resolving issues around antipsychotic 